\title{
Percepção de risco para infecções sexualmente transmissíveis em idosos
}

\author{
Risk perception for sexually transmissible infections in elderly \\ Percepción de riesgo para infecciones sexualmente transmisibles en ancianos
}

Apolo Kassio Barros da Silva ORCID: https://orcid.org/0000-0003-0526-6169 Centro Universitário de Ciências e Tecnologia do Maranhão, Brasil E-mail:apolo19972009@gmail.com

Antonio Vinícius Barros da Silva ORCID: https://orcid.org/0000-0002-0137-9409 Centro Universitário de Ciências e Tecnologia do Maranhão, Brasil E-mail: barrosvinicius719@gmail.com Karine Costa Melo ORCID: https://orcid.org/0000-0001-8253-859X Universidade Federal do Maranhão, Brasil E-mail: karinemelo09@gmail.com

Darci Rosane Costa Freitas Alves ORCID: https://orcid.org/0000-0001-7839-9996

Universidade Estadual do Maranhão, Brasil E-mail: darci_rane@ hotmail.com

E-mail: darci_rane@ hotmail.com
Wygor Bruno e Silva Morais ORCID: https://orcid.org/0000-0001-5219-0465 Centro Universitário de Ciências e Tecnologia do Maranhão, Brasil E-mail: wygorleao@hotmail.com

Paulo Ricardo Viana de Carvalho ORCID: https://orcid.org/0000-0002-2138-9903

Universidade Estadual do Maranhão, Brasil E-mail: prcarvalho585@gmail.com

\begin{abstract}
Resumo
Objetivo: Avaliar a percepção de risco para infecções sexualmente transmissíveis em idosos. Método: Trata-se de um estudo transversal, do tipo descritivo e exploratório, com abordagem quantitativa dos dados desenvolvida em Centros de Convivência de Idosos (CCI) da cidade Caxias-MA, foram incluídos na pesquisa 220 participantes. Houve a aplicação de questionário semiestruturado em forma de entrevista. Resultados: Cerca de 72,3\% dos idosos eram do sexo feminino, com média de idade de 71,44 anos, viúvos (45,5\%); que possuem parceiro afetivo (48,6\%) sexualmente ativos (31\%); que se satisfaziam com sexo com penetração (26\%); onde $41,8 \%$ não usavam preservativo embora $87,3 \%$ não se consideram em risco de contrair qualquer IST. Conclusão: O comportamento sexual dos idosos torna os idosos vulneráveis, na evidencia de conhecimento deficitário sobre infecções sexualmente transmissíveis (IST), bem como a prática sexual insegura fazendose necessário criação de estratégias de conscientização para promoção de um envelhecimento saudável e vencimento dos preconceitos existentes.
\end{abstract}

Palavras-chave: Idosos; Sexualidade; Doenças sexualmente transmissíveis.

\section{Abstract}

Objective: To assess the perception of risk for sexually transmitted infections in the elderly. Method: This is a cross-sectional study, descriptive and exploratory, with a quantitative approach to data developed in Community Centers for the Elderly (CCI) in the city of Caxias-MA, 220 participants were included in the research. There was the application of a semistructured questionnaire in the form of an interview. Results: About $72.3 \%$ of the elderly were female, with a mean age of 71.44 years, widowed (45.5\%); who have an affective partner (48.6\%) who are sexually active (31\%); who were satisfied with penetrative sex $(26 \%)$; where $41.8 \%$ did not use condoms although $87.3 \%$ do not consider themselves at risk of contracting any STI. Conclusion: The sexual behavior of the elderly makes the elderly vulnerable, in the evidence of deficient knowledge about sexually transmitted infections (STIs), as well as unsafe sexual practice, making it necessary to create awareness strategies to promote healthy aging and overcoming prejudices existing.

Keywords: Aged; Sexuality; Sexually transmitted diseases.

\section{Abstracto}

Objetivo: Evaluar la percepción de riesgo de infecciones de transmisión sexual en ancianos. Método: Se trata de un estudio transversal, descriptivo y exploratorio, con un abordaje cuantitativo de los datos desarrollados en los Centros Comunitarios de Ancianos (CCI) de la ciudad de Caxias-MA, se incluyeron 220 participantes en la investigación. Se aplicó un cuestionario semiestructurado en forma de entrevista. Resultados: Alrededor del 72,3\% de los ancianos eran mujeres, con una edad media de 71,44 años, viudos (45,5\%); que tienen una pareja afectiva (48,6\%) que es sexualmente activa (31\%); que estaban satisfechos con el sexo con penetración (26\%); donde el $41,8 \%$ no utilizó preservativo aunque el $87,3 \%$ no se considera en riesgo de contraer alguna ITS. Conclusión: El comportamiento sexual de las personas mayores hace que las personas mayores sean vulnerables, ante la evidencia de un conocimiento deficiente sobre las infecciones de transmisión sexual (ITS), así como una práctica sexual insegura, por lo que es necesario crear estrategias de concienciación para promover un envejecimiento saludable y la superación de los prejuicios existentes.

Palabras clave: Anciano; Sexualidad; Enfermedades de transmisión sexual.

\section{Introdução}

Nas últimas décadas, houve um aumento substancial da população idosa impulsionada pelo aumento da expectativa de vida, a queda na taxa de natalidade, urbanização, melhores condições alimentares, higiene pessoal e ambiental e desenvolvimento de novas tecnologias nos serviços de saúde tem possibilita a cura e controle de diversas patologias (Dornelas Neto et al., 2015). 
Segundo a Organização Pan Americana de Saúde - OPAS, (2005) o envelhecimento é um processo individual, acumulativo, não patológico e irreversível de deterioração de um organismo maduro, comum a indivíduos de toda espécie de maneira que o tempo o faça capaz de fazer frente aos estresses do meio ambiente, aumentando, portanto, sua possibilidade de morte.

Considerando as conquistas dos idosos ao longo dos anos, o prolongamento da vida sexual é fator de destaque, pois com a melhoria da qualidade de vida e criação de novas tecnologias na área da saúde; além do desenvolvimento de produtos farmacêuticos, observa-se uma vida sexual ativa entre os idosos na atualidade. No entanto, essa prática sexual do idoso, aliada a diversos fatores intrínsecos e extrínsecos da própria identidade de ser idoso; muitas vezes torna-se insegura, o que acaba por contribuir para o surgimento de Infecções Sexualmente Transmissíveis - IST, (Dornelas Neto et al., 2015). Tal realidade, aponta desafios a serem enfrentados pela família, serviços de saúde e sociedade em geral (Cezar et al., 2012).

Dentre a relação das IST's e a prática sexual insegura do idoso, apresenta-se o conceito de vulnerabilidade que, na área da Saúde Pública que foi associado a epidemia de HIV/AIDS na década de 1990. Contudo, o conceito de vulnerabilidade se apresenta como um campo ainda a ser explorado; passível de elaboração conceitual capaz de alcançar um amplo e heterogêneo universo de reflexões e práticas (Malagon; Dina, 2015). Para a epidemiologia, a vulnerabilidade é compreendida como um movimento que considera a chance de exposição das pessoas ao adoecimento, como resultante de um conjunto de aspectos individuais, coletivos, contextuais que acarretam maior suscetibilidade à infecção e ao adoecimento (Rosa et al., 2012).

A vulnerabilidade do idoso às infecções sexualmente transmissíveis, engloba um amplo aspecto de interrelações entre sexo, faixa etária, escolaridade, estado civil, proveniência, renda, vida sexual ativa, orientação sexual, uso de preservativos, conhecimento sobre infecções sexualmente transmissíveis e o uso de medicações que melhoram o desempenho sexual (Silva et al., 2018).

Frente a essa problemática tem-se a seguinte questão norteadora: Qual a percepção de risco do idoso para infecções sexualmente transmissíveis (IST)?

Para tal, elencaram-se o seguinte objetivo de avaliar a percepção de risco para infecções sexualmente transmissíveis em idosos e identificar em idosos fatores de vulnerabilidade às infecções sexualmente transmissíveis na perspectiva de sua percepção de risco.

\section{Metodologia}

Trata-se de uma pesquisa de campo do tipo descritiva exploratória, com abordagem quantitativa dos dados.

Segundo Gerhardt e Silveira, (2009), a pesquisa de caráter exploratória se caracteriza como o tipo de pesquisa que busca uma maior familiaridade com o tema, com o objetivo de traze-los mais à mostra; enquanto a pesquisa descritiva se caracteriza como a pesquisa que visa descrever determinados aspectos, fenômenos e fatos de uma dada realidade.

A abordagem quantitativa dos dados permite por meio de cálculos matemáticos a explicação do fenômeno e relação entre variáveis, proporcionando uma maior representatividade à pesquisa pois geralmente a amostra constitui-se de número consideravelmente bom e se dá por meio do uso de instrumentos padronizados e neutros, os resultados da pesquisa são interpretados como um retrato real da população estudada (Gerhart \& Silveira, 2009).

A pesquisa foi realizada em seis Centros de Convivência para Idosos (CCI's) na cidade de Caxias - MA. Participaram da pesquisa 220 idosos de ambos os sexos com idade igual ou superior a 60 anos. A coleta de dados ocorreu no primeiro semestre de 2019 (janeiro a abril) por meio de um questionário semiestruturado e padronizado com perguntas fechadas e abertas, no qual para questões fechadas os participantes da pesquisa poderiam escolher apenas uma opção de resposta e para questões abertas poderiam citar mais de uma resposta. Entre os riscos que a pesquisa oferecia aos participantes estava o constrangimento e desconforto em relação ao tema a ser explorado e as perguntas investigadas via questionário. Contudo cabe dizer que foram realizadas orientações pontuais quando aos objetivos da pesquisa; quanto a anonimato dos participantes. Firma-se que a entrevista foi realizada em ambiente privativo e/ou o próprio participante poderia responder ao questionário sem intermédio do pesquisador, caso assim preferisse. Além disso, o pesquisado foi orientado quanto a possibilidade de recusar responder quaisquer questões e desistir da pesquisa a qualquer tempo.

Os resultados foram tabulados utilizando-se o programa Microsoft Office Excel versão 2010 para Windows e as análises estatísticas através do SPSS versão 20.0 para Windows, no qual foram calculadas: média, mínima, máxima. O nível de significância adotado para este estudo foi de $\mathrm{p}<0,05$. Esta pesquisa faz parte do projeto guarda-chuva denominado "Comportamento sexual e prevenção de Infecções Sexualmente Transmissíveis em centros de convivências de idosos" com aprovação do comitê de ética em pesquisa com o número CAAE: 97090918.2.0000.8007, conforme preconiza a Resolução nº 466/2012 do CNS/MS. 


\section{Resultados}

Entre os 220 idosos participantes, 159 (72.3\%) eram do sexo feminino e 61 (27.7\%) do sexo masculino, com idade variando entre 60 a 91 anos e idade média de 71.44 anos. Houve prevalência do sexo feminino na faixa etária de 60-65 anos (26.4\%). Observouse ainda, que 54 participantes (24.5\%) eram solteiros, 64 (29.1\%) casados, 36 (16.4\%) divorciados e 67 (30.5\%) viúvos.

Os participantes foram questionados quanto a vivência de sua sexualidade; assim, $48.6 \%$ dos idosos se auto classificaram sexualmente ativos e 50\% sexualmente inativos. Quanto a forma de satisfação em suas relações íntimas observou-se que $30.9 \%$ dos idosos se satisfaziam com sexo com penetração; $12.2 \%$ com carícias e $6.4 \%$ com beijos. A masturbação não foi citada pelos participantes. Cerda de $45.5 \%$ dos idosos entrevistados possuíam parceiro afetivo e grande maioria dos idosos (75.9\%) não se relacionam com múltiplos parceiros, conforme mostra Tabela 1.

Tabela 1 - Distribuição do comportamento sexual na vivência da sexualidade dos idosos e o modo pelo qual se satisfazem em seus relacionamentos íntimos. $(\mathrm{n}=220)$.

\begin{tabular}{lcc}
\hline Variáveis* & N & \% \\
\hline Prática da sexualidade & & \\
Ativa & 107 & 48.6 \\
Inativa & 110 & 50 \\
Não respondeu & 3 & 1.4 \\
Satisfação sexual & & \\
Sexo com penetração & 68 & 30.9 \\
Carícias & 27 & 12.2 \\
Beijos & 14 & 6.4 \\
Masturbação & 0 & 0.0 \\
Não se aplica/Não Respondeu & 111 & 50.5 \\
Parceiro Afetivo & & \\
Sim & 100 & 45.5 \\
Não & 120 & 54.5 \\
Múltiplos Parceiros & & \\
Sim & 53 & 24.1 \\
Não & 167 & 75.9 \\
TOTAL & 220 & 100 \\
\hline
\end{tabular}

*Os participantes poderiam citar mais de uma resposta. IST=infecções sexualmente transmissíveis; $\mathrm{N}=$ número de quantidade; $\%=$ percentil. Fonte: Pesquisa direta.

Quanto ao uso de preservativos, cerca de $41.8 \%$ dos idosos não faziam uso e apenas $5.5 \%$ usavam sempre o método preventivo. Na avaliação de auto percepção de risco para IST's, houve prevalência de idosos que não assumiam risco de adquirir qualquer infecção sexualmente transmissível (87.3\%), evidenciando um déficit de auto percepção de risco, conforme evidencia a Tabela 2.

Tabela 2 - Distribuição da prática do uso de preservativos nas relações sexuais e auto percepção de risco dos idosos. (n=220).

\begin{tabular}{lcc}
\hline Variáveis & N & \% \\
\hline Uso do preservativo (camisinha) & & \\
Sim, sempre & 12 & 5.5 \\
Não, nunca & 92 & 41,8 \\
Algumas vezes & 6 & 2.7 \\
Não se aplica/não responderam & 110 & 50.0 \\
\hline Auto percepção de risco para IST & & \\
Sim & 28 & 12.7 \\
Não & 192 & 87.3 \\
\hline
\end{tabular}

IST=infecções sexualmente transmissíveis; $\mathrm{N}=$ número de quantidade; $\%=$ percentil. Fonte: Pesquisa direta.

No presente estudo, observou-se que $83.6 \%$ dos idosos entrevistados não haviam sido acometidos por IST's. Entre os que já haviam sido infectados com alguma IST (15.9\%) destacou-se a sífilis (6.4\%); linfogranuloma venéreo (0.9\%), gonorreia (4.5\%) e linfogranuloma venéreo (0.9\%), (Tabela 3$)$. 
Tabela 3 - Histórico e distribuição das infecções sexualmente transmissíveis nos idosos participantes da pesquisa. (n=220).

\begin{tabular}{lcccc}
\hline Variáveis & & \multicolumn{3}{c}{ TOTAL } \\
\hline Histórico de IST & $\mathrm{N}$ & $\%$ & $\mathrm{~N}$ & $\%$ \\
Sim & 35 & 15,9 & & \\
Não & 184 & 83,6 & 220 & 100 \\
Não respondeu & 1 & 0,5 & & \\
\hline IST* & & & $\mathrm{N}$ & $\%$ \\
Sífilis & 14 & 6,4 & 35 & 100 \\
Linfogranuloma venéreo & 2 & 0,9 & 35 & 100 \\
Gonorreia & 10 & 4,5 & 35 & 100 \\
Não lembra & 12 & 5,5 & 35 & 100 \\
\hline
\end{tabular}

* Os participantes poderiam citar mais de uma resposta. IST=infecções sexualmente transmissíveis; $N=$ número de quantidade; $\%=$ percentil; Fonte: Pesquisa direta.

Tabela 4 - Correlação entre as variáveis fixas e o comportamento de risco do idoso para ISTs. Caxias - MA, 2019.

\begin{tabular}{lcccccccc}
\hline \multirow{2}{*}{ Variáveis } & \multicolumn{2}{c}{ Sexo } & \multicolumn{2}{c}{ Idade } & \multicolumn{2}{c}{ Parceiro fixo } & \multicolumn{2}{c}{ Usa Preservativo } \\
\cline { 2 - 10 } & C.C. ${ }^{\#}$ & P*** & C.C. ${ }^{*}$ & P*** & C.C. ${ }^{*}$ & P*** & C.C. ${ }^{*}$ & P*** $^{* * *}$ \\
\hline Conhece métodos preventivos & -0.040 & 0.557 & 0.058 & 0.392 & -0.046 & 0.497 & -0.037 & 0.587 \\
Considera-se com risco para IST & 0.129 & 0.056 & 0.072 & 0.290 & -0.090 & 0.185 & $0,175^{* *}$ & 0.009 \\
Já teve ou tem alguma IST & $0,206^{* *}$ & 0.002 & -0.095 & 0.162 & -0.061 & 0.370 & $0,178^{* *}$ & 0.008 \\
\hline
\end{tabular}

Legenda: $*(<0,05) ; * *(<0,01) ; * * *$ Spearman's Correlation; C.C.\# = Coeficiente de correlação. Fonte: Pesquisa Direta (2018).

Tratando-se da população da amostra como um todo, analisando as variáveis fixas e o comportamento sexual dos idosos constatou-se associações significativa entre as variáveis: Já teve ou tem alguma IST e Sexo $(p=0,002)$, e o Uso do preservativo $(p=$ $0,08)$. A variável, considera-se com risco para IST com o Uso do preservativo $(p=0,009)$.

Tabela 5 - Correlação entre sujeitos sexualmente ativos com a percepção de risco para desenvolver IST’s. Caxias - MA, 2019.

\begin{tabular}{|c|c|c|c|c|c|c|c|c|}
\hline \multirow[b]{2}{*}{ Variáveis } & \multicolumn{2}{|c|}{ Sexo } & \multicolumn{2}{|c|}{ Idade } & \multicolumn{2}{|c|}{ Parceiro fixo } & \multicolumn{2}{|c|}{ Usa Preservativo } \\
\hline & C.C.\# & $\mathrm{P}^{* * * *}$ & C.C.\# & $\mathrm{P} * * *$ & C.C.\# & $\mathrm{P}^{* * *}$ & C.C.\# & $\mathrm{P} * * *$ \\
\hline Conhece métodos preventivos & -.042 & .671 & .073 & .455 & .116 & .236 & .007 & .945 \\
\hline Já teve ou tem alguma IST &, $327 * *$ & .001 & -.068 & .484 & .045 & 648 &, $203 *$ & .036 \\
\hline
\end{tabular}

Legenda: $*(<0,05) ; * *(<0,01) ; * * *$ Spearman's Correlation; C.C.\# = Coeficiente de correlação. Fonte: Pesquisa Direta (2018).

Entres os participantes autodeclarados sexualmente ativos e suas percepções de risco encontra-se associações significativas entre as variáveis: Sexo e já teve ou tem alguma IST $(\mathrm{p}=0,001)$, considera-se com risco para IST $(\mathrm{p}=0,04)$; já teve ou tem alguma IST com o Uso do preservativo $(\mathrm{p}=0,03)$.

\section{Discussão}

No que diz respeito a vulnerabilidade do idoso às infecções sexualmente transmissíveis, Silva et al. (2018) aponta que o sexo, faixa etária, escolaridade, estado civil, proveniência, renda, vida sexual ativa, orientação sexual, uso de preservativos, conhecimento sobre infecções sexualmente transmissíveis e o uso de medicações que melhoram o desempenho sexual são fatores considerados associados e determinantes de risco.

Estudos realizados no Nordeste apontam que os idosos têm se mantido sexualmente ativos e que a prática de sexo regular acontece de forma insegura deixando-os vulneráveis as infecções sexualmente transmissíveis (Andrade et al., 2017).

O uso do preservativo ainda é a forma mais segura de se prevenir infecções sexualmente transmissíveis (IST), no qual protege cerca de $80 \%$ a $95 \%$ contra as chances de contrair alguma IST, entretanto a adesão ao método ainda é baixa (Thiesen, 2018).

Os achados referentes aos resultados de auto percepção de risco e uso de preservativo apresentam similaridade com os estudos realizados por Brito et al. (2016), com 55 idosos em duas unidades básicas de saúde, onde 40\% dos idosos citaram como método de prevenção, o uso do preservativo, porém $76.4 \%$ declaram não estar sob risco de contrair alguma infecção sexualmente 
transmissíveis (IST) tais fatores contribuem para que fiquem vulneráveis o que pode resultar no aumento do número de idosos infectados por IST.

Observa-se que os motivos que levam ao não uso do preservativo são o fato da gravidez não ser mais um risco; a carência de informação, por achar que estão nos últimos anos de sua vida, não sendo um problema adquirir uma doença nesta fase da vida ou pelas dificuldades que possam surgir com o uso do preservativo tais como, dificultar a ereção e desempenho sexual e/ou a diminuição da sensibilidade; o direcionamento de ações de educação em saúde exclusivamente para o público jovem e isso influência diretamente na auto percepção de risco e como desfecho final o número de infecções sexualmente transmissíveis tende a aumentar (Castro et al., 2014).

Estudos apontam que o aumento da expectativa de vida, bem como os avanços nas ações de saúde, a prática sexual insegura torna os idosos susceptíveis a adquirir infecções sexualmente transmissíveis, diante do estigma da sexualidade do idoso, ações de prevenção são direcionadas única e exclusivamente aos público jovem e adulto-jovem (Burigo et al., 2015).

A sífilis é uma doença que assim como outras infecções sexualmente transmissíveis podem ser transmitidas de diversas formas, porém a forma mais frequente é por contato sexual desprotegido ou verticalmente pela mãe na gestação (Kalinin,2016). O simples fato de uma parcela ter apresentado alguma IST ao longo da vida indica presença de comportamento de risco para reinfecção ou quadro novo de infecção evidencia vulnerabilidade individual (Andrade et al., 2017).

Corroborando com os dados aqui apresentados, estudo realizado por Dornelas Neto et al. (2018) com objetivo de analisar a tendência evolutivas de IST em idoso no Brasil, revelou que as IST que mais tem causado problemas e de maior incidência são a HIV/AIDS são a Clamídia, Sífilis, Gonorreia, Condiloma acuminado e Herpes simples, estas se destacam em países como a Austrália, Estados Unidos, China, Canadá, Coréia, Países Africanos. No Brasil os dados especificamente voltados ao idoso são escassos devido a subnotificação.

Estudo realizado por Pimenta, Moraes e Sousa, (2015), numa análise sobre a prevalência do HIV/AIDS nas regiões do Brasil verificou-se que houve um aumento na prevalência de idoso infectados cerca de 7.884 casos na qual a região Sudeste apresentou maior índice com 3.437 casos em cinco anos; Sul em seguida com 1.974 caos e em terceiro lugar o Nordeste com 1.386 casos registrados.

É imprescindível que o enfermeiro enquanto autor da educação em saúde quebre tabus e estimule campanhas e discussões acerca do tema e então evitar dupla carga de doenças (IST + comorbidades), pois o que se nota é que os idosos não estão preparados para essa realidade de sua sexualidade visto que seus conhecimentos acerca dos perigos são escassos (Venturini et al, 2018).

\section{Conclusão}

Considerando o aumento da expectativa de vida, bem como a longevidade da vida social e vivência da sexualidade por parte dos idosos, embora o processo de senescência traga consigo algumas limitações os idosos têm se adaptado bem as mudanças, ainda que em grande parte a vivência da sexualidade ocorra de forma insegura. Dessa forma conclui-se que o comportamento sexual dos idosos para prevenção de infecções sexualmente transmissíveis, baseado na vivência da sexualidade e no déficit na autopercepção de risco, torna o idoso vulnerável a infecções sexualmente transmissíveis.

Faz-se necessário ações de abordagem sobre sexualidade à população idosa visando a conscientização desta população pautada no princípio da promoção da saúde e prevenção de agravos à saúde.

\section{Referências}

Andrade, J. et al. (2017). Vulnerability of the elderly to sexually transmitted infections. Acta Paulista de Enfermagem. 30 (1), 8-15. https://doi.org/10.1590/19820194201700003

Brito, N. M. I.de. et al. (2016). Idosos, infecções sexualmente transmissíveis e AIDS: conhecimentos e percepção de risco. ABCS Health Sciences. 41 (3). https://doi.org/10.7322/abcshs.v41i3.902.

Burigo, G. da F. et al. (2015). Sexualidade e comportamento de idosos vulneráveis a doenças sexualmente transmissíveis. CuidArte, Enferm. 9 (2), 148-153. ID: biblio1027922 .

Castro, S. de F. F. de. et al. (2014). Prevenção da AIDS em idosos: visão e prática do enfermeiro. Ciência \& Saúde. 7 (3), 131-140. https://doi.org/10.15448/1983652X.2014.3.17773.

Cezar, A. K., Aires, M., \& Paz, A. A. (2012). Prevenção de doenças sexualmente transmissíveis na visão de idosos de uma Estratégia da Saúde da Família. Rev. bras. enferm. [online]. 65 (5), 745-750. ISSN 0034-7167. http://dx.doi.org/10.1590/S0034-71672012000500005. Acesso em: 23/02/2018.

Dornelas Neto, J.et al. (2015). Doenças sexualmente transmissíveis em idosos: uma revisão sistemática. Ciência \& Saúde Coletiva. 20, 3853-3864. https://doi.org/10.1590/1413-812320152012.17602014.

Kalinin, Y. (2016). Sífilis: aspectos clínicos, transmissão, manifestações orais, diagnóstico e tratamento. Odonto. 23 (45-46), 65-76. https://doi.org/10.15603/21761000/odonto.v23n45-46p65-76

Malagon Ora., \& Dina C. (2015). O conceito de vulnerabilidade e seu caráter biossocial. Interface. (53), 237-250. http://dx.doi.org/10.1590/1807-57622014.0436. 
OPAS. Organização Mundial da Saúde. (2005). Envelhecimento ativo: uma política de saúde. Organização Pan-Americana da Saúde.

Pimenta, C. J. L., Moraes, J. C. O., \& Sousa, E. de. (2015). Prevalência de HIV/AIDS em idosos entre 2010 e 2014 no Brasil. Cieh., 2 (1). https://www.editorarealize.com.br/editora/anais/cieh/2015/TRABALHO_EV040_MD2_SA2_ID2220_26072015153622.pdf.

Rosa, F. et al. (2012). Prevalência de anti-HCV em uma população privada de liberdade. Rev Assoc Med Bras. 58 (5), 557-560. http://ramb.elsevier.es/pt/prevalenciaanti-hcv-em-uma-populacao/articulo/90159988/.

Silva, J. D. B. et al. (2018). Vulnerabilidade às Infecções Sexualmente Transmissíveis/ AIDS em idosos. Revista uningá, 53 (1), http://revista.uninga.br/index.php/uninga/article/view/1418>. Acesso em: 05/05/2018.

Thiesen, F. (2018). Ist's e uso de preservativos. Revista UNIPLAC. 6 (1).

Venturini, Larissa et al. (2018). Atuação da equipe de enfermagem frente à sexualidade de idosas institucionalizadas. Revista da Escola de Enfermagem da USP. 52, 03302. https://doi.org/10.1590/S1980-220X2017017903302. 